\title{
1 Death recognition by undertaker bees
}

2 Wen Ping ${ }^{1,2 *}$

31 CAS Key Laboratory of Tropical Forest Ecology, Xishuangbanna Tropical Botanical Garden,

4 Chinese Academy of Sciences, Mengla, Yunnan Province, 650223, China

52 Center for Plant Ecology, Core Botanical Gardens, Chinese Academy of Sciences, Mengla,

6 Yunnan 666303, China

*Correspondence: wenping@xtbg.ac.cn

Abstract: Dead conspecifics removal is important of being social to avoid pathogen transmission, which resulted in the evolution of a specific caste of undertaking workers in all hives bee species. However, it is mysterious that how the undertakers distinguish death and life instantly. Through integrative studies of behavioural tests and chemical analyses, a novel mechanism for dead conspecifics recognition is found in the Asian bee Apis cerana cerana Fabricius. The bees detect quickly the death of conspecifics based on decreased cuticular hydrocarbon (CHC) emissions, caused by the cooling of the dead bee. Specifically, with the decline of body temperature in death, the CHC emission was reduced. Undertakers perceived the major CHCs. Addition of synthetic CHCs, followed by heating, inhibited undertaking behaviour. Among these CHCs, heptacosane and nonacosane are the major compounds in a natural bee hive, providing a continuous signal associated with life. Via changing the vapour pressure then the ratio of emitted compounds encoding the physiological status of signal sender, insect chemical communication can be finely tuned by body temperature. This straightforward death recognition mechanism requiring little cost can be universal in animal living in social groups, especially in the social insects. Body temperature affected behaviour can response to increasing frequency of extreme weathers in global climate change, which help explain the recent worldwide bee health problem.

Keywords: life signal, cuticular hydrocarbon, insect body temperature, vapour pressure, GC-EAD, social behaviour 


\section{Introduction}

Recognition of death is an important behaviour of being social. In social groups, detection of death and rapid removal of corpses is essential in order to defend the colony against pathogen transmission (1-4). Humans use the cessation of heartbeats and breathing or cooling body temperature to diagnose death. All these even require complicated processing of information due to the development of resuscitation skills. However, what mechanisms are available to much more primitive organisms such as insects to assess whether conspecifics are alive or dead? It has been a long time, that carbonic acids derived from corpse decompose are responsible for the death recognition of dead bodies in social insects $(2,5)$. But this leads to theoretical and empirical conflicts. Theoretically, dead individuals, usually old or diseased workers, are at the highest risk of carrying pathogens. Leaving dead bodies not removed till decomposition increased the risk of pathogen transmission within the nest, which is conflicted with the importance of corpse management in social behaviour. In observations, specialized workers in eusocial species perform undertaking behaviour, removing the dead bodies of workers speedily (3, 6-8). Usually, a dead bee can be recognized within $30 \mathrm{~min}$ (7), before significant decomposition occurs with the concomitant production of odours of decay. In the darkness of a bee hive, no visual cues are available, and tactile cues from a freshly dead bee are probably indistinguishable from those of a living bee. Thus, how do undertaking workers recognize dead bees?

Chemical cues specific to life or death could signal rapidly death in social insects (3, 5). For example, in ants, it has been shown that the decrease in chemical signals is correlated with death. Two specific chemical compounds associated with life, dolichodial and iridomyrmecin, were identified in the Argentine ant, Linepithema humile, and these compounds inhibited necrophoresis behaviour (8). Also, the dead worker of termite Reticulitermes flavipes produce 3-octanone, 3-octanol as death signal (9). In honeybee, the blend of $\beta$-ocimene and oleic acid acts as a cue for diseased brood in hygienic behaviour of Apis mellifera (10). But it is not known if there is any compound responsible for recognizing the death of workers.

The dynamics of emission of a signal are related to the signal's behavioural function in a social context. For a recruitment signal, release should be positively 
correlated with the number of individuals involved $(11,12)$. In contrast, a decrease in inhibitory signals can result in the release of an inhibited behaviour. Based on the correlation between the dynamics of signal emission and the behavioural process being affected, signals mediating certain behaviours can be identified, for example in the identification of recruitment signals in termite foraging (13), honeybee alarm communication (14) and most sex pheromone in calling moth (15). In contrast to the collective decision making by a large number of workers in swarming behaviour (16, 17), undertaking bees are a small subset of individuals in the hive. The behavioural process of undertaking does not require the involvement of large numbers of individuals, so recruitment signal is not likely to exist for the recognition of dead bees. Also, undertaking bees can be experienced in the removal process (18), but there is no appetitive reward or aversive punishment to undertaking bees that may generate the associative learning of olfactory cues. Thus, the perception of chemical cues associated with life and death is likely to be innate (7).

Cuticular hydrocarbons (CHCs) have multiple functions in social insect communication $(19,20)$. They typically consist of long chain hydrocarbons with more than 20 carbons, characterized by low vapour pressure, and function as low-volatility signals in short distance or contact communication (21). Most known queen pheromones or reproductive-specific signals in wasps, bumble bees, ants, and termites are CHCs (22-24). Even behavioural polymorphism within a caste is regulated by CHCs, for example in Pogonomyrmex barbatus (20). Besides the Nasonov pheromone, CHCs are the major cuticular compounds in honeybee (25), functioned as colony discrimination signals (26-30) and even behavioural polymorphism signals (31). In waggle dance communication, the detection of CHCs can stimulate followers (32). Besides signalling, CHCs have additional roles in providing physical protection of the colony. For example, CHCs are major components in the wax comb as building materials (33). Also, CHCs are abundant on the body surface of most insects, serving to maintain water balance. The persistence of waxy CHCs due to their low vapour pressure and stability is suited to these physiological functions (34). Individuals thus need to balance the two roles of releasing CHCs for short distance communication, while also producing and retaining CHCs in larger quantities for desiccation resistance. In honeybee, besides contact cues, short distance communication can be signalled by semivolatile CHCs; for example, CHC headspace volatiles detected in the waggle dance area are different from those in other areas of the nest (32). 
Social insects can discriminate CHCs. In Camponotus ants, single sensillum recording (SSR) and learning and memory tests showed that a broad spectrum of CHCs can be detected by the olfactory neurons but with precise discrimination (35). Honeybees can also perceive and discriminate among different CHCs in learning and memory assays $(36,37)$. But it was not directly shown if honeybees can discriminate CHCs physiologically or not.

Living honeybees have body temperatures ranging from $35{ }^{\circ} \mathrm{C}$ to $45{ }^{\circ} \mathrm{C}$ (38-40), usually higher than the hive wall temperature under ambient conditions. When a bee dies, the decrease in body temperature reduces the vapour pressure of its CHCs, decreasing their emission. This might have helped the chemical signalling in nestmate recognition (38). Thus, rather than using decreasing body temperature as a death signal, honeybees may perceive the reduction of CHCs in the headspace around a dead individual as a death signal. Along these lines, Visscher (1983) showed that addition of paraffin (containing many of the alkanes found in honeybee CHCs) inhibited necrophoresis behaviour in Apis mellifera, and exhaustive extraction by Soxhlet extraction also lead to failure of death recognition (7). With increasing frequency of extreme weather events in recent years (41), any temperature based behaviour can response to climate changes. But till now it is not known how bee health was affected by climate change directly.

Till now, no chemical life and/or death signal was identified in honeybees, and little on this was known in most social insects. By knowing chemical cues for death recognition, we can assess the complexity and diversity of this widely distributed behaviour in social insects, which helps explain why social behaviour can be evolved multiple times from different lineages. Here, my objective was to identify the signals used to detect death in the Asian honeybee, Apis cerana. By using headspace volatile collection, gas chromatography (GC), and coupled gas chromatography-mass spectrometry (GC-MS), I compared the volatiles from living and dead bees. I analysed the effect of body temperature of living bees on the evaporation of CHCs using thermal imaging and simulation. I tested the antennal perception of bees to specific CHCs, using coupled GC-electroantennography (GC-EAD). Then I performed reduction and addition bioassays with CHCs, testing the hypothesis that the decrease of CHC emissions signalled the reduction of body temperature as a specific signal eliciting honeybee undertaking behaviour. 


\section{Results}

In performing undertaking behaviours, undertaking bees would palpate a dead bee, check around it several times and then directly grab the dead bee and attempt to fly away. Dead bees were dropped at distances away from the colony in observations. Basically, the dead bees were recognised by their reduced cuticular hydrocarbon emissions (Fig 1.).

\section{Life signal is cuticular}

8 Bioassays of worker body sections showed that body parts were removed selectively (GLMM: $G^{2}{ }_{7}=56.375, P<0.001$ ); the more cuticle on the body parts, the more they were removed (Sig $=P<0.05$ ). The stings with alarm pheromone as controls were always ignored $(P=0.74>0.05)$. Despite the abundant volatiles emitted as alarm pheromones, the stings are not removed more than clean filter paper controls, which excluded the possibility of using alarm pheromones as a signal of life (Fig. 2A).

\section{Effect of heating and/or washing on undertaking}

Active living bees on the floor area had an average body temperature of $43.6 \pm 1.7$ ${ }^{\circ} \mathrm{C}$ (mean \pm SD, $t$-test: $t=0.018, d f=164, P=0.99$ ), whereas dead bees cooled to ambient temperature $\left(15{ }^{\circ} \mathrm{C}\right.$ to $25{ }^{\circ} \mathrm{C}$ during tests) soon after perishing. By using 3 equal sized colonies, no effect from colony (GLMM: $G_{2}^{2}=0.00, P=1.0$ ) or colony*dead bee treatments interaction (GLMM: $G^{2}{ }_{10}=4.11, P=0.942$ ) was observed on the ratio of dead bees removed. The effect of dead bee treatments with heating, cooling and body wash at different times on the ratio of dead bees removed was significant (GLMM: $G_{5}^{2}=65.04, P<0.001$ ). Fresh dead bees were all rapidly removed under natural ambient temperatures in $0.5 \mathrm{~h}$. In contrast, heated dead bees were removed only after much longer periods (up to 2 h) or if they were allowed to cool to ambient temperature (Fig. 2B). Also, washed bees deprived of most of their CHCs and heated were always removed (EM: $88.0 \pm 4.2 \%, n=72)$ more than heated unwashed dead bees (EM: $0.0 \pm 0.0 \%, \mathrm{n}=72, P<0.001$ ), but as much as long-time (3h) heated dead bees (EM: $76.0 \pm 5.2 \%, n=72 P=0.144>0.05)$ (Fig. 2B). These 
two results suggested that cuticular signals affected by body temperature might be regulating undertaking behaviour.

\section{CHC emission diminished on dead bees}

HS-SPME-GC analysis revealed that CHC emissions decreased after death in Apis cerana. Twelve peaks were significantly higher in the headspace volatiles of living bees than in dead bees (paired t-test: $n=9$, sig $=P<0.05$ ) (Fig. 2C). Nine of these compounds were CHCs, including heneicosane (C21) $(t=9.99, d f=8, P<0.001)$, tricosane (C23) ( $t=11.28, d f=8, P<0.001)$, (Z)-7-pentacosene (C25:1) $(t=2.74, d f$ $=8, P=0.026)$, (Z)-9-pentacosene (C25:1) $(t=4.80, d f=8, P=0.001)$, pentacosane (C25) ( $t=4.88, d f=8, P=0.001)$, (Z)-9-heptacosene (C27:1) $(t=3.20, d f=8, P=$ 0.013), heptacosane (C27) ( $t=4.76, d f=8, P=0.001)$, nonacosenes (C29:1) $(t=4.04$, $d f=8, P=0.004)$ and nonacosane (C29) $(t=3.37, d f=8, P=0.010)$. GC-EAD analyses showed that undertaking bees could detect all the major CHCs, including C23, C25, C27, and C29, and (Z)-11-eicosenyl acetate, a contaminant from venom (Fig. 2D). Among the four CHCs, C27 and C29 generated the largest responses.

\section{Vapour pressure linked body temperature and $\mathrm{CHC}$ emission}

Vapour pressure calculations showed that within the body temperature changes, C27:1, C27, C29:1 and C29 had the biggest changes in vapour pressure (Fig. 2E). The vapour pressure controlled the evaporation, and consequently the quantities of CHCs in the headspace volatiles. Thus, the evaporated CHCs, especially C27 and C29, were reduced substantially between live and dead bees.

\section{Synthetic CHCs inhibits undertaking under active body temperature}

In assays of the synthetic CHCs, undertaking bees checked the CHC-modified dead bees continuously until they decided to carry the dead bees away. From the durations recorded, there were significant effects from the colony (GLM: $F_{(4,362)}=15.50, P<$ $0.001)$, the environmental temperature $\left(F_{(13,362)}=2.03, P=0.018<0.05\right)$, the tested samples $\left(F_{(12,362)}=6.50, P<0.001\right)$, the interactions of colony*samples $\left(F_{(19,362)}=\right.$ 2.58, $P<0.001)$ and the interaction of temperature*samples $\left(F_{(50,362)}=1.94, P<\right.$ 
0.001). Generally, the larger the colony, the faster they removed the dead bees $(P<$ 0.05), and the undertaking behaviour was inhibited best when ambient temperature was $25{ }^{\circ} \mathrm{C}(P<0.05)$. Extracted dead bees tested at ambient temperature were removed quickly (EM: $6.7 \pm 4.25 \mathrm{~min}, \mathrm{n}=26)$. Addition of C21 (EM: $33.5 \pm 4.21 \mathrm{~min}$, $\mathrm{n}=30)$, C27 (EM: $37.3 \pm 3.88 \mathrm{~min}, \mathrm{n}=33$ ), C29 (EM: $36.3 \pm 3.64 \mathrm{~min}, \mathrm{n}=52)$, and a mixture of CHCs (EM: $41.2 \pm 5.06 \mathrm{~min}, \mathrm{n}=20$ ) to extracted and heated bees significantly inhibited undertaking behaviour $(P<0.05)$ (Fig. 2G). Also, treatment of dead bees with wax solution also inhibited undertaking, with a significant effect of the quantities of wax added to the number of dead bees removed (GLMM: $G^{2}{ }_{2}=12.723$, $P=0.002<0.05)$. The more wax added, the stronger the inhibition $(P<0.05)$.

\section{Discussion}

\section{Body temperature-vapour pressure correlation in bee $\mathrm{CHC}$ emissions}

Results suggest that the bee death is encoded by the reduction of $\mathrm{CHC}$ emissions caused by the cooling of a dead bee on the hive floor. Undertakers apparently do not use the decrease of body temperature as a life signal (see Fig 2 B,G Washed but heated bodies were removed quickly.), even though they have thermo receptors (42) and may use it in nestmate recognition (38). Instead, undertakers use the associated reduction of $\mathrm{CHC}$ volatiles as an indication of death. On dead bees, the CHCs retained on the body surface as contact cues do not change within the short time when undertaking behaviour occurs (Fig. S3). Undertaking bees appear to use the decrease of headspace CHC volatiles (emitted CHCs) to discriminate living or dead bees. Due to the low vapour pressure of CHCs, researches on CHCs in contact communication has used solvent extraction or SPME fibre wiping to sample CHCs on the body surface, but not the actual amount of CHCs in the headspace that may function in short range communication. Short-distance headspace volatile collection can explain more precisely the role of CHCs emission in insect communication.

Ratios of signal compounds encode behavioural specific information in social insects $(11,13)$. Although SPME is not accurate in detecting the actual ratios of CHC compounds, basically, the ratio of compounds in the volatiles is quite different from the ratio of compounds in the whole body extract under the same HS-SPME extraction method (Fig. 2C, D). In headspace samples, C27:1 and C29:1 were the 
major compounds, whereas in whole body extracts, the saturated alkanes C23, C25, C27, and C29 were the major compounds. The calculation of vapour pressures predicted that the volatilities of C27:1, C27, C29:1 and C29 would be most affected by variations in body temperature. This could explain the ratio differences, but I cannot exclude the possibility that $\mathrm{C} 27: 1$ and C29:1 in the headspace volatiles were from glands or internal tissues with smaller cuticular area (43). The relatively higher vapour pressure of $\mathrm{C} 21$, makes it the major emitted $\mathrm{CHC}$ in the headspace around a bee, although its quantities on body surfaces were relatively low compared to other compounds. Coincidentally, C21 is the reproductive-specific signal in the termite Reticulitermes flavipes (23). In calculations, the alkenes C27:1 and C29:1 showed the biggest changes in volatility with body temperature, but they were not significantly active as signals of life. Instead, bees attracted to the artificially warmed dead bees with added C27:1 and C29:1 samples performed balling behaviour (44). However, these compounds are only present in trace amounts on the body surface (See FID of whole body extract in Fig. 2D), so it is unlikely that they would be reliable signals of life. Further tests are needed to show whether these compounds are involved in defensive behaviours of honeybees.

Body temperature of active insects are always higher than environmental temperature, for example in singing bladder cicadas (45), flying sphinx moth (46). Body temperature variations between physiological status such as active and quiescent conditions are sure to affect greatly the vapour pressure of pheromone compounds secreted from body surface. And it is common for insect to use hydrocarbons of different molecule size for communication (15). Major CHCs studied here were differed by 2 carbons between, resulting in highly varied ratios of the compounds emitted according to the changing body temperature under different physiological status (see calculation results in Fig. S4). Since ratios of compounds are always encoding species-specific or physiological and behavioural specific information, by considering the body temperature affected ratio of volatiles, more details can be explicated in insect chemical communication, especially in the social insect communication, which is mediated mostly by CHCs.

\section{Precise perception to CHCs in communication}

In GC-EAD tests, all CHCs lacking an oxygen-containing group elicited weak 
responses $(47,48)$. Thus, EAD systems with a high sensitivity amplifier $(14,49)$ and static noise control (filtering the air flow with copper mesh and a stainless steel shield cage) were used in this research. The setup enabled the first EAD recording of honeybee responses to CHCs. The biased EAD responses to different CHCs indicated the discrimination ability of olfactory receptors. This can be further investigated with SSR tests. Moreover, processing of signals in the brain, leading to behavioural outputs, can be controlled by neural hormones (50), so that the peripheral perception of compounds may not determine the behavioural responses. Thus, comparative recordings of neural responses to CHCs in undertakers, foragers, and guards may help explain the behavioural polymorphism in honeybee workers. Based on clear discrimination of compounds, with C23:1, C23, and C25 encouraging waggle dance followers in Apis mellifera (32), and C21, C27, and C29 encoding life status in A. cerana found here, the differentiation of the behavioural function of $\mathrm{CHC}$ indicates again the complex chemical communication system in honeybee hives that is still relatively poorly understood.

By considering the effect of body temperature, CHC-based honeybee communication can be explained in more detail. In the complex background odour, the use of variable ratios of headspace CHCs could alleviate signal interference for more precise communication. Using thermal images of hives, detailed maps of CHC signalling can help explain much clearer the communication in various behaviours.

\section{Bee health response to climate change via affected body temperature}

Here, temperature dropping scale affects the change of CHC emissions, allowing for recognition of dead bees and triggering of undertaking behaviours. Extreme weather conditions can affect the dropping scale of body temperature from alive to dead and weak bees in the hive. With increasing frequency of extreme weather events in recent years (41), the pathogen defence by undertaking and hygienic behaviour can be affected. Using the decrease of CHC signals to trigger undertaking behaviour can be inclusive. In extremely cold days, the cold anesthetized and/or weak bees may also be recognized and removed by undertakers (51) in the mornings. If not warm up, those cold anesthetized bees taken out of the hive will die. Also, in extremely hot days, hives may be subjected to high temperatures more often than previously, possibly increasing the chance of pathogen transmission through dead bee contact, via failure 
to recognize the dead or weak bees warmed up unusually. Here, together with the fact that temperature experience of brood affect the behaviour of adult bees (52), honey bees may response to the increasing frequency of extreme weather with high sensitivity, resulted in colony collapse disorder.

For beekeeping practice, with honeybee pathogens such as Nosema (53) and Varroa mite transmitted viruses (54) spreading worldwide, causing great losses in global apiculture, the optimization of undertaking and hygienic behaviour can help improve the resistance to pathogens. Understanding the chemical communication in undertaking behaviour provides a basis for developing methods for optimizing honeybee undertaking and hygienic behaviour. Thus, lowering the hive floor temperatures, for example, by adding a ventilated bottom panel to hives, may help the recognition of infected bees based on this research.

\section{Straightforward death diagnosis may help the evolution of sociality}

Along the line of related researches (5), no compound of unique pheromone structure is needed for instant death recognition. Also, the CHCs signal for life in honeybees can be of same as CHCs used by solitary species in stress resistance, parasitism, aggression, competition and/or predation (34). Compared with the ants and termites using specific more volatile life signal $(5,8)$, the body size may affect the strategies used. The climbing tiny ants and termites are less likely to be warmed up to a much higher temperature than the ambient condition, which limited the chance of using CHCs for multi-purpose. But the ant and termite life and death compounds were also used in other behaviours. Thus, specifity of life or death signal can be simply quantitative not necessarily qualitative. Undertaking or necrophoresis, the important step of pathogen defence, is crucial for the evolution of higher sociality. Hereby, social species should spend neither more energy in evolving immunity with undertaking against pathogens transmission, nor more energy in evolving a new life signal for undertaking than solitary species. Simply, straightforward death recognition makes no more difficulty to become social safely.

\section{Supplementary information}

Material and methods and supplementary figures and table were provided in 
1 Supplementary information. Crude data for figures were provided in the ZIP files.

2

3

4

5

\section{Acknowledgements}

I thank Prof. Jocelyn G. Millar in UCR, Prof. Jin Chen and Prof. Zhanqi Chen in XTBG and Prof. James Nieh in UCSD for critic comments and edits. I am grateful to Lu Zi-Yun, Yang Zhen-Xin, Na Bo, Klett Katrina and Zhao Qin, for their help in bioassays. I thank Lin Hua in XTBG for kindly providing infrared camera. I acknowledge the Public Technology Service Centre of XTBG for providing help on instruments.

\section{References}

1. Anderson James R, Biro D, Pettitt P. Evolutionary thanatology. Philosophical Transactions of the Royal Society B: Biological Sciences. 2018;373(1754):20170262.

2. Wilson EO, Durlach NI, Roth LM. Chemical releasers of necrophoric behavior in ants. Psyche. 1958;65(4).

3. Sun Q, Zhou X. Corpse management in social insects. Int J Biol Sci. 2013;9(3):313-21.

4. Kesäniemi J, Koskimäki JJ, Jurvansuu J. Corpse management of the invasive Argentine ant inhibits growth of pathogenic fungi. Sci Rep-Uk. 2019;9(1):7593.

5. Sun $Q$, Haynes KF, Zhou X. Managing the risks and rewards of death in eusocial insects. 2018;373(1754):20170258.

6. Robinson GE, Page RE. Genetic determination of guarding and undertaking in honey-bee colonies. Nature. 1988;333(6171):356-8.

7. Visscher PK. The honey bee way of death: Necrophoric behaviour in Apis mellifera colonies. Anim Behav. 1983;31(4):1070-6.

8. Choe D-H, Millar JG, Rust MK. Chemical signals associated with life inhibit necrophoresis in Argentine ants. Proceedings of the National Academy of Sciences. 2009;106(20):8251.

9. Sun $Q$, Haynes KF, Zhou X. Dynamic changes in death cues modulate risks and rewards of corpse management in a social insect. Funct Ecol. 2017;31(3):697-706.

10. McAfee A, Chapman A, lovinella I, Gallagher-Kurtzke Y, Collins TF, Higo H, et al. A death pheromone, oleic acid, triggers hygienic behavior in honey bees (Apis mellifera L.). Sci Rep-Uk. 2018;8(1):5719.

11. Wen X-L, Wen P, Dahlsjö CAL, Sillam-Dussès D, Šobotník J. Breaking the cipher: ant eavesdropping on the variational trail pheromone of its termite prey. Proceedings of the Royal Society B: Biological Sciences. 2017;284(1853).

12. Jackson DE, Ratnieks FLW. Communication in ants. Curr Biol. 2006;16(15):R570-R4.

13. Wen $\mathrm{P}, \mathrm{Ji} \mathrm{B}-\mathrm{Z}$, Sillam-Dussès $\mathrm{D}$. Trail communication regulated by two trail pheromone 
components in the fungus-growing termite Odontotermes formosanus (Shiraki). Plos One. 2014;9(3):e90906.

14. Wen P, Cheng Y-N, Qu Y-F, Zhang H-X, Li J-J, Bell H, et al. Foragers of sympatric Asian honey bee species intercept competitor signals by avoiding benzyl acetate from Apis cerana alarm pheromone. Sci Rep-Uk. 2017;7(1):6721.

15. Allison JD, Cardé RT. Pheromone communication in moths: evolution, behavior, and application. Allison JD, Cardé RT, editors. Oakland: University of California Press; 2016.

16. Visscher PK, Camazine S. Collective decisions and cognition in bees. Nature. 1999;397:400.

17. Visscher PK. Group decision making in nest-site selection among social insects. Annu Rev Entomol. 2006;52(1):255-75.

18. Trumbo ST, Robinson GE. Learning and task interference by corpse-removal specialists in honey bee colonies. Ethology. 1997;103(11):966-75.

19. Howard RW, Blomquist GJ. Ecological, behavioral, and biochemical aspects of insect hydrocarbons. Annu Rev Entomol. 2004;50(1):371-93.

20. Greene MJ, Gordon DM. Cuticular hydrocarbons inform task decisions. Nature. 2003;423:32.

21. Leonhardt SD, Menzel F, Nehring V, Schmitt T. Ecology and evolution of communication in social insects. Cell. 2016;164(6):1277-87.

22. Van Oystaeyen A, Oliveira RC, Holman L, van Zweden JS, Romero C, Oi CA, et al. Conserved class of queen pheromones stops social insect workers from reproducing. Science. 2014;343(6168):287-90.

23. Funaro $\mathrm{CF}$, Böröczky K, Vargo EL, Schal C. Identification of a queen and king recognition pheromone in the subterranean termite Reticulitermes flavipes. Proceedings of the National Academy of Sciences. 2018;115(15):3888.

24. Conte YL, Hefetz A. Primer Pheromones in Social Hymenoptera. Annu Rev Entomol. 2007;53(1):523-42.

25. Francis BR, Blanton WE, Nunamaker RA. Extractable surface hydrocarbons of workers and drones of the genus Apis. J Apicult Res. 1985;24(1):13-26.

26. Rahman S, Hajong SR, Gévar J, Lenoir A, Darrouzet E. Cuticular hydrocarbon compounds in worker castes and their role in nestmate recognition in Apis cerana indica. J Chem Ecol. 2016;42(5):444-51.

27. Page RE, Metcalf RA, Metcalf RL, Erickson EH, Lampman RL. Extractable hydrocarbons and kin recognition in honeybee (Apis mellifera L.). J Chem Ecol. 1991;17(4):745-56.

28. Breed MD, Garry MF, Pearce AN, Hibbard BE, Bjostad LB, Page JRE. The role of wax comb in honey bee nestmate recognition. Anim Behav. 1995;50(2):489-96.

29. Slessor KN, Winston ML, Le Conte Y. Pheromone communication in the honeybee (Apis mellifera L.). J Chem Ecol. 2005;31(11):2731-45.

30. D'Ettorre P, Wenseleers T, Dawson J, Hutchinson S, Boswell T, Ratnieks FLW. Wax combs mediate nestmate recognition by guard honeybees. Anim Behav. 2006;71(4):773-9.

31. Francis BR, Blanton WE, Littlefield JL, Nunamaker RA. Hydrocarbons of the cuticle and hemolymph of the adult honey bee (Hymenoptera: Apidae). Ann Entomol Soc Am. 1989;82(4):486-94. 32. Thom C, Gilley DC, Hooper J, Esch HE. The scent of the waggle dance. Plos Biol. 2007;5(9):e228. 33. Fröhlich B, Tautz J, Riederer M. Chemometric classification of comb and cuticular waxes of the honeybee Apis mellifera carnica. J Chem Ecol. 2000;26(1):123-37.

34. Blomquist GJ, Nelson DR, De Renobales M. Chemistry, biochemistry, and physiology of insect cuticular lipids. Arch Insect Biochem. 1987;6(4):227-65. 
35. Sharma Kavita R, Enzmann Brittany L, Schmidt Y, Moore D, Jones Graeme R, Parker J, et al. Cuticular hydrocarbon pheromones for social behavior and their coding in the ant antenna. Cell Reports. 2015;12(8):1261-71.

36. Getz WM, Smith KB. Olfactory sensitivity and discrimination of mixtures in the honeybeeApis mellifera. Journal of Comparative Physiology A. 1987;160(2):239-45.

37. Châline N, Sandoz J-C, Martin SJ, Ratnieks FLW, Jones GR. Learning and discrimination of individual cuticular hydrocarbons by honeybees (Apis mellifera). Chem Senses. 2005;30(4):327-35. 38. Stabentheiner $A$, Kovac $H$, Schmaranzer $S$. Honeybee nestmate recognition: the thermal behaviour of guards and their examinees. J Exp Biol. 2002;205(17):2637.

39. Fuchikawa T, Shimizu I. Effects of temperature on circadian rhythm in the Japanese honeybee, Apis cerana japonica. J Insect Physiol. 2007;53(11):1179-87.

40. Stabentheiner A, Vollmann J, Kovac $H$, Crailsheim K. Oxygen consumption and body temperature of active and resting honeybees. J Insect Physiol. 2003;49(9):881-9.

41. Stott $P$. How climate change affects extreme weather events. Science. 2016;352(6293):1517.

42. Junca P, Sandoz J-C. Heat Perception and Aversive Learning in Honey Bees: Putative Involvement of the Thermal/Chemical Sensor AmHsTRPA. Front Physiol [Internet]. 2015 2015; 6:[316 p.].

43. Wang Q, Goodger JQD, Woodrow IE, Elgar MA. Location-specific cuticular hydrocarbon signals in a social insect. Proceedings of the Royal Society B: Biological Sciences. 2016;283(1827):20160310.

44. Ono M, Igarashi T, Ohno E, Sasaki M. Unusual thermal defense by a honeybee against mass attack by hornets. Nature. 1995;377(6547):334-6.

45. Josephson RK, Young D. Body temperature and singing in the bladder cicada, Cystosoma saundersii. J Exp Biol. 1979;80(1):69.

46. Heinrich B. Temperature regulation of the sphinx moth, Manduca sexta. J Exp Biol. 1971;54(1):141.

47. Chen G, Wang Z-W, Wen P, Wei W, Chen Y, Ai H, et al. Hydrocarbons mediate seed dispersal: a new mechanism of vespicochory. New Phytologist. 2018;15166(0).

48. Saïd I, Gaertner C, Renou M, Rivault C. Perception of cuticular hydrocarbons by the olfactory organs in Periplaneta americana (L.) (Insecta: Dictyoptera). J Insect Physiol. 2005;51(12):1384-9.

49. Wen P, Cheng Y-N, Dong S-H, Wang Z-W, Tan K, Nieh JC. The sex pheromone of a globally invasive honey bee predator, the Asian eusocial hornet, Vespa velutina. Sci Rep-Uk. 2017;7(1):12956.

50. Schulz DJ, Barron AB, Robinson GE. A Role for octopamine in honey bee division of labor. Brain, Behavior and Evolution. 2002;60(6):350-9.

51. Döke MA, Frazier M, Grozinger CM. Overwintering honey bees: biology and management. Current Opinion in Insect Science. 2015;10:185-93.

52. Tautz J, Maier S, Groh C, Rössler W, Brockmann A. Behavioral performance in adult honey bees is influenced by the temperature experienced during their pupal development. Proceedings of the National Academy of Sciences. 2003;100(12):7343.

53. Klee J, Besana AM, Genersch E, Gisder S, Nanetti A, Tam DQ, et al. Widespread dispersal of the microsporidian Nosema ceranae, an emergent pathogen of the western honey bee, Apis mellifera. J Invertebr Pathol. 2007;96(1):1-10.

54. Wilfert L, Long G, Leggett HC, Schmid-Hempel P, Butlin R, Martin SJM, et al. Deformed wing virus is a recent global epidemic in honeybees driven by Varroa mites. Science. 2016;351(6273):594. 
bioRxiv preprint doi: https://doi.org/10.1101/2020.03.05.978262; this version posted March 5, 2020. The copyright holder for this preprint (which was not certified by peer review) is the author/funder, who has granted bioRxiv a license to display the preprint in perpetuity. It is made available under aCC-BY-NC-ND 4.0 International license.

\section{Figure}

2
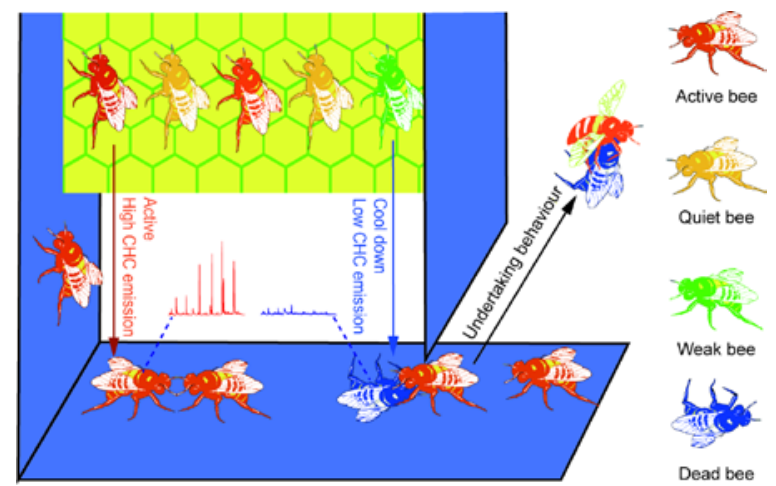

3 Figure 1 Undertaking behaviour is triggered by low CHC emission

4 Bees are coloured according to their status and body temperature as shown in the right side. Active bees were warm in red,

5 while dead bees were cool in blue. Hive parts are also coloured in the similar pattern. The comb is warm in yellow, while 6 the hive walls are cool in blue.

7

8 
A

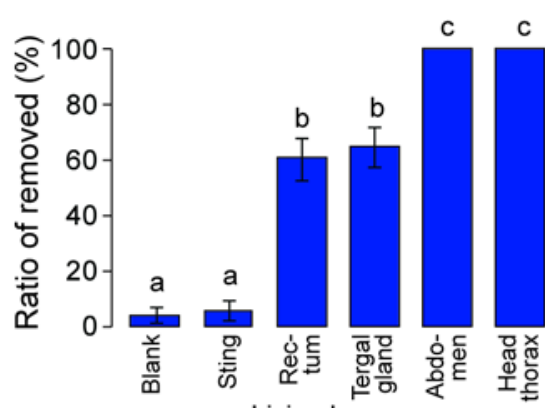

C

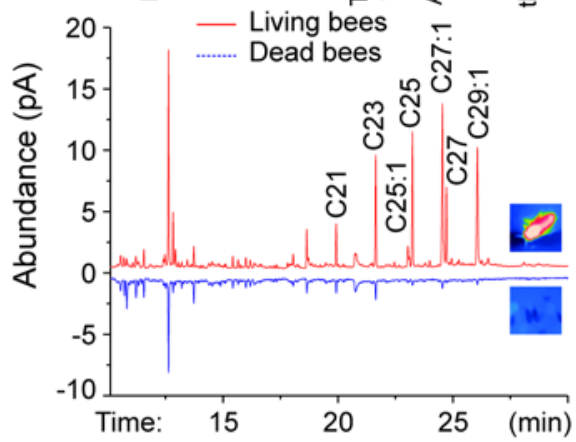

E

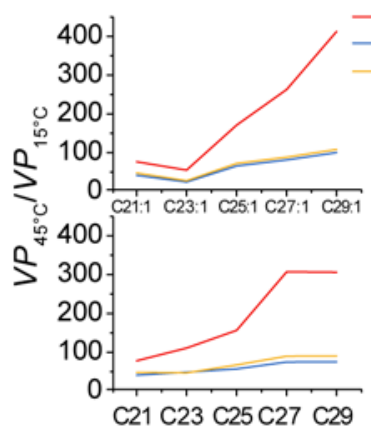

C21 C23 C25 C27 C29
- Antoine
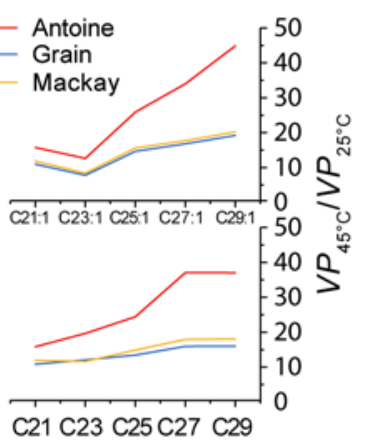
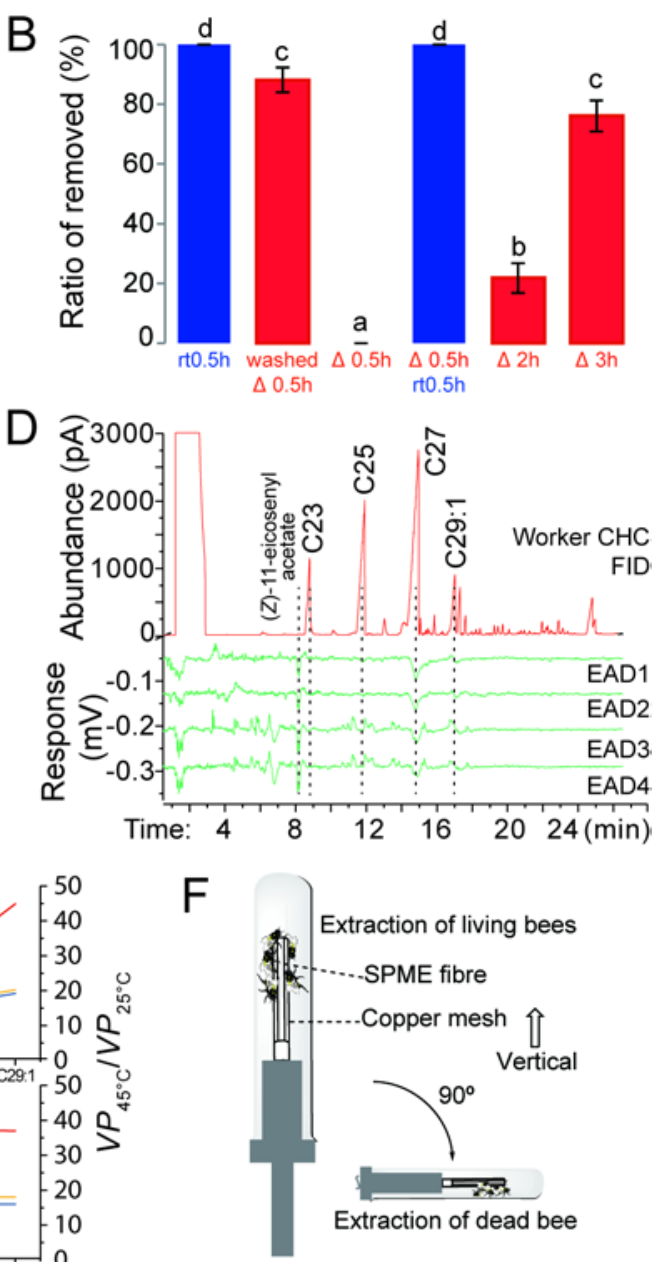

HS-SPME for $\mathrm{CHC}$
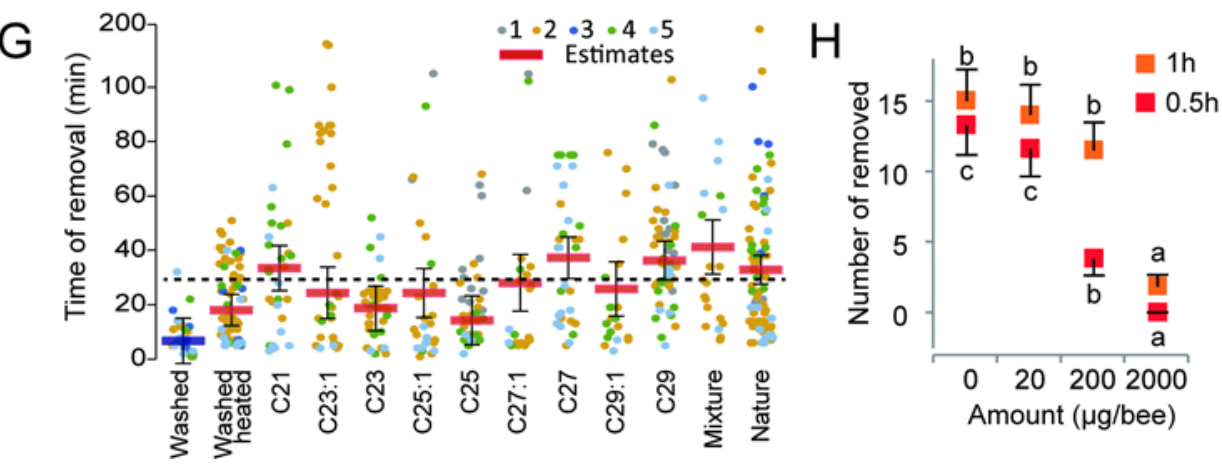

Figure 2 Honeybees perceive death from the reduction of CHC emission

A) Localization of death recognition signals to the cuticle. Blank: a filter paper without any body parts; Abdomen: the abdominal cuticle. Columns with different letters were significantly different at the $P<0.01$ level. B) Effect of heating on undertaking behaviour. Column and samples are coloured in red for heated $(\Delta)$ and in blue for ambient temperature (rt). Columns with different letters are significantly different at the $P<0.01$ level. C) Comparative analyses of the HS-SPME extraction of cuticular volatiles between living and dead bees. D) GC-EAD analysis for the perception of CHCs. E) Changes of $\mathrm{CHC}$ vapour pressure at different body temperatures of living $\left(45^{\circ} \mathrm{C}\right)$ and dead bees $\left(15{ }^{\circ} \mathrm{C} \sim 25{ }^{\circ} \mathrm{C}\right)$. F) Diagram for HS-SPME collection of bee volatiles at close range. G) Undertaking inhibition bioassay for CHC addition and reduction on dead bees. Columns higher than the dashed line are significantly different (Sig $=P<0.05$ ) from the washed bees tested at ambient temperature as control. Mixture is the mixture of synthetic CHCs as described in methods. Nature is heated dead bee. Different colours of points represent different colonies. Columns are coloured in red for heated and in blue for ambient temperature. H) Undertaking inhibition bioassay by adding wax solution to dead bees. Columns 
bioRxiv preprint doi: https://doi.org/10.1101/2020.03.05.978262; this version posted March 5, 2020. The copyright holder for this preprint (which was not certified by peer review) is the author/funder, who has granted bioRxiv a license to display the preprint in perpetuity. It is made available under aCC-BY-NC-ND 4.0 International license.

1

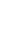

marked with different letters are significantly different (Sig $=P<0.05$ ). Data recorded successively at $0.5 \mathrm{~h}$ (error bar downward) and $1 \mathrm{~h}$ (error bar upward) were analysed separately but plotted together. 


\section{Supplementary Information}

\section{Death recognition by undertaker bees}

3 Wen Ping ${ }^{1,2 *}$

41 CAS Key Laboratory of Tropical Forest Ecology, Xishuangbanna Tropical Botanical Garden,

5 Chinese Academy of Sciences, Mengla, Yunnan Province, 650223, China

62 Center for Plant Ecology, Core Botanical Gardens, Chinese Academy of Sciences, Mengla,

7 Yunnan 666303, China

$8 \quad *$ Correspondence: wenping@xtbg.ac.cn

\section{$9 \quad$ Materials and methods}

\section{Bees}

The five A. cerana cerana Fabricius colonies used in this experiment were maintained

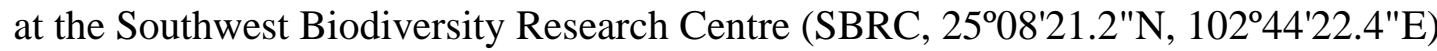
in Kunming, Yunnan, using standard beekeeping protocols. Natural nectar and pollen resources were available in the local habitat through the year. Observation hives were set up in a working shed at the same place. Workers which picked up dead bees were classified as undertaking bees. The five colonies were not always uniform in size, having varied number of individuals (Table S1).

\section{Localization of death recognition signal}

Foragers collected in front of the hive were killed by freezing at $-40{ }^{\circ} \mathrm{C}$ for $10 \mathrm{~min}$. These fresh dead bees were then dissected into different parts, including the rectum, the sting apparatus, the tergal glands, the head and thorax and the remaining abdominal cuticle. All these body parts were subjected to bioassay within 10 min after dissection. In bioassays, each body part was positioned on a clean filter paper disk (1.0 cm diameter, cleaned by soaking in pure water). To emit odour from internal tissue or glands, the head with mandibular glands and the rectum were squashed on the filter papers. All samples were positioned on the floor of observation hives. Tests 
were done under ambient hive conditions. Bioassays were carried out on sunny autumn days when colonies were relatively inactive. In $2 \mathrm{~h}$, the number of samples removed and the total number of samples tested were recorded as event/trail data. Samples selectively picked up and then dumped outside the hive were considered to contain the signal for dead body discrimination.

\section{Living bee body temperature measuring}

To confirm the difference between the temperature of living and dead bees, a T1040 infrared camera (FLIR, Oregon, USA) was used to record high definition thermal images and video at the bottom of an observation hive. In this floor area where dead bees drop, there were usually moving living bees as well, often foragers coming in and out. The temperature of living bees on the recorded hive floor area was measured using FLIR tools software (FLIR, Oregon, USA).

\section{Inhibitory heating bioassays}

Because the reduction in headspace CHCs was recognized by undertaking bees, the effect of reducing body temperature on CHC emission was tested. I placed fresh dead bees as prepared above for whole body bioassays on two different glass Petri dishes (9 cm id) (Fig. S1), with one held at ambient temperature, and the other heated using a custom-made controller, composed of a 9 cm glass Petri dish, a W1209 thermostatic controller (Telesky, Shenzhen, CN), a 10 K MF58 thermistor (Risym, Shenzhen, CN) and a 15 W Cr20Ni80 heating wire (Yusheng, Jiangsu, CN). After calibration using the T1040 infrared camera (Fig. S1), in the dish, bees were heated to a steady temperature, approximating that of a living bee $\left(38 \sim 41^{\circ} \mathrm{C}\right)$. Temperatures inside and outside the hive were measured using a type K thermocouple read by a VC97 digital multimeter (Victor, Shanghai, CN). In the tested hives, the difference in average body temperature between a dead bee and a living bee was $10 \sim 25{ }^{\circ} \mathrm{C}$. In treatments, dead bees which were not removed were considered as inhibiting undertaking behaviour.

\section{$\mathrm{CHC}$ reduction and addition bioassays}

Because the assays above suggested that CHCs were associated with the death 
signal, the effects of reduction and addition of CHCs were tested. Bees were freeze-killed as stated above, and the stings and venom sacs were removed so as not to agitate the bees in the hive. I removed the stings because the experiments above had shown that they were not active in necrophoresis, but would activate the hive, hampering undertaking behaviour. In CHC reductions, fresh dead bees were washed three times with $3 \times 0.5 \mathrm{~mL}$ hexane in a $1.5 \mathrm{~mL}$ glass pipette, and then were taken out and dried for 10 min to evaporate the solvent. By washing with a small volume of solvent instead of Soxhlet extraction, the CHCs on the surface of the dead bee were not eliminated, but only reduced to about one fifth of original amount according to the GC analyses of the concentrated first and second washes of the same bees (Fig. S2). In CHC addition, three types of sample were prepared: (I) all single synthetic compounds were dissolved in hexane at $10 \mathrm{mg} / \mathrm{mL}$; (II) for three isomers of each alkene, each isomer was added respectively at $6.6 \mu \mathrm{L}$ (i.e., $66 \mu \mathrm{g} / \mathrm{bee}$ ); (III) to simulate the natural $\mathrm{CHC}$ profile, a mixture of $8 \mathrm{mg} \mathrm{C} 21,3 \mathrm{mg}$ C23:1s (Z5:Z7:Z9=1:1:1), 60 mg C23, 3 mg C25:1s (Z5:Z7:Z9=1:1:1), 70 mg C25, 3 mg C27:1s (Z5:Z7:Z9=1:1:1), 90 mg C27, 3 mg C29:1s (Z5:Z7:Z9=1:1:1), and $60 \mathrm{mg}$ C29 were dissolved in $10 \mathrm{~mL}$ hexane. Extracted bees were dosed with $20 \mu \mathrm{L}$ of these CHC solutions (i.e., $200 \mu \mathrm{g} / \mathrm{bee}$ ). After addition, bees were dried and warmed for 5 $\min$ at $40{ }^{\circ} \mathrm{C}$ to evaporate the solvent, then introduced to the floor of the beehive. Bees were tested in groups of 3 to 6 per trial based on the activity of the hive (estimated by number of bees collected within $5 \mathrm{~min}$ at the hive entrance). Three extracted dead bees under ambient conditions and three extracted and heated dead bees were used as controls in all tests. The time between the test sample introduction and removal was recorded for each test bee. The numbers of replicates were decided by the numbers of workers that can be collected within $5 \mathrm{~min}$ in front of each tested hive on each day, which is affected by the size of the hive.

Major compound of bee wax are esters at $71 \%$, the major one is triacontanyl palmitate (C46H92O2). Others are alcohol and acids. The ratio varies according to origin and species. CHCs are $\sim 14 \%$ that can be volatile in normal temperature, other major compounds are esters with 30 more carbons that is not volatile $(1,2)$. In another experiment, comb wax containing all the major CHC components was dissolved in hexane at $1 \mathrm{mg} / \mathrm{ml}, 10 \mathrm{mg} / \mathrm{mL}$, or $100 \mathrm{mg} / \mathrm{mL}$. Fresh dead bees were first washed as described above. Then the bees were dosed with $20 \mu \mathrm{L}$ of the wax solutions, to make wax-coated dead bees with $20 \mu \mathrm{g}, 200 \mu \mathrm{g}$, or $2000 \mu \mathrm{g}$ per bee. The wax-coated bees 
were dried and heated as described above to remove solvent. Both fresh $\mathrm{CHC}$ reduced and added bees were placed in a $9 \mathrm{~cm}$ petri dish in the bee hive for undertaking bioassays. Each wax dose was replicated 15 times. Three colonies were tested. Natural dead bees were used as control. The numbers of removed and tested bees were recorded for each concentration in each colony.

\section{Collection of volatiles from living and dead bees}

To analyse the difference in CHC emissions between living and dead bees, the CHCs evaporated from bodies over short distances were compared using headspace solid phase micro-extraction (HS-SPME). Living bees were collected from three colonies. In each colony, 5 bees were collected without disturbance and then held in the dark in a clean glass test tube (18 mm id, $180 \mathrm{~mm}$ long). A $65 \mu \mathrm{m}$ PDMS/DVB SPME fibre (Supelco, CA) protected by a copper mesh tube (40 mesh, $3 \mathrm{~mm}$ id, 50 mm long) was used to collect volatiles emitted by the bees. When extracting living bees, the apparatus was kept upright. Bees would keep moving upward to gather near the SPME fibre. The living bees were extracted in darkness first. I collected headspace volatiles from the living bee in the dark to reduce bee movement. Then, these bees were frozen and killed; their stings were removed using a pair of forceps as described above for the fresh dead bee samples. The dead bees were then placed near the SPME fibre. All the samples were extracted for $0.5 \mathrm{~h}$ under ambient conditions, with bees contacting the copper mesh but not the SPME fibre, to obtain the cuticular volatiles.

\section{Chemical analysis}

\section{Chemical standards}

Commercially available heneicosane, (Z)-9-tricosene (97\%, in GC-FID peak area hence force), tricosane (99\%), and pentacosane (99\%) were purchased from TCI (City, Japan). Other CHCs, including (Z)-5-tricosene (98\%), (Z)-7-tricosene (97\%), (Z)-5-pentacosene (97\%), (Z)-7-pentacosene (98\%), (Z)-9-pentacosene (97\%), (Z)-5-heptacosene (99\%), (Z)-7-heptacosene (97\%), (Z)-9-heptacosene (97\%), (Z)-5-nonacosene (99\%), (Z)-7-nonacosene (97\%), and (Z)-9-nonacosene (97\%), were 
synthesized following literature procedures (3). Briefly, terminal alkynes (1.0 eq) were deprotonated using 1M n-BuLi (1.05 eq) in hexane in dry THF/HMPA (2:1) at $-70{ }^{\circ} \mathrm{C}$ for $30 \mathrm{~min}$, then coupled to alkyl bromides $(1.0 \mathrm{eq})$ at $-70{ }^{\circ} \mathrm{C}$ to room temperature (rt) for $4 \mathrm{~h}$. The alkynes were then reduced to cis-alkenes using $\mathrm{H}_{2}$ and Lindlar catalyst $\left(5 \% \mathrm{Pd} / \mathrm{BaSO}_{4}\right)$ in the presence of quinolene in hexane at rt. Heptacosane (99\%) and nonacosane (99\%) were prepared from further reduction of corresponding alkenes using hydrogen with 5\% $\mathrm{Pd} / \mathrm{C}$ catalyst in hexane at room temperature. All products were purified by vacuum removal of semi-volatile impurities and then silica gel chromatography, eluting with hexane.

\section{GC-MS and GC-EAD}

An HP7890-5975C (Agilent, Palo Alto, USA) GC-MS system was used with 1 $\mathrm{mL} / \mathrm{min}$ helium as carrier gas. The splitless inlet with a small inner diameter liner for SPME samples (Supelco, Bellefonte PA, USA) was heated to $250{ }^{\circ} \mathrm{C}$. An HP-5ms (30 $\mathrm{m} \times 250 \mu \mathrm{m} \times 0.25 \mu \mathrm{m}$ film, Agilent, Palo Alto, USA) column was used. The oven ramp was set as $50{ }^{\circ} \mathrm{C}$ for $1 \mathrm{~min}$ and then $10^{\circ} \mathrm{C} / \mathrm{min}$ to $280{ }^{\circ} \mathrm{C}$ for $10 \mathrm{~min}$. The transfer line was $250{ }^{\circ} \mathrm{C}$. In the quadrupole mass spectrometer, a $70 \mathrm{eV}$ EI ion source was used, heated to $230{ }^{\circ} \mathrm{C}$. The mass range scanned was $\mathrm{m} / \mathrm{z} 28.5-500$ at $4 \mathrm{scan} / \mathrm{s}$. The abundance threshold for detection was set to 10. Data were collected and analysed using MSD Chemstation software (Agilent Technologies, Palo Alto CA, USA). Based on the literature $(4,5)$, all compounds were identified by comparing their retention times and mass spectra to those of the synthetic standards.

For GC analyses, an HP7890B GC (Agilent) was used to separate the HS-SPME extracts with $1.0 \mathrm{~mL} / \mathrm{min}$ helium as carrier gas. The splitless inlet was heated to 260 ${ }^{\circ} \mathrm{C}$. An HP-5 $(30 \mathrm{~m} \times 320 \mu \mathrm{m} \times 0.32 \mu \mathrm{m}$, Agilent $)$ column was used. The oven ramp was set as $50{ }^{\circ} \mathrm{C}$ for $1 \mathrm{~min}$ and then $10{ }^{\circ} \mathrm{C} / \mathrm{min}$ to $280{ }^{\circ} \mathrm{C}$ for $10 \mathrm{~min}$. FID signals were recorded and analysed using Chemstation software. Analyses of HS-SPME-GC were replicated 9 times (3 colonies each by 3 times).

I used a custom-made GC-EAD and EAG system $(6,7)$ to test the antennal electrophysiological responses of undertaking bees. The cuticle with two antennal bases of each undertaking bee was dissected from the head capsule with a scalpel. The preparation was mounted to the reference electrode by surface tension of the Ringer's solution $^{6}$. The tips of the antennae were cut off using iris scissors under a microscope. 
1 The tips of each of the two antennae were mounted to two LMP7721 (Texas Instruments, Dallas, TX, USA) based probes using glass electrodes filled with Ringer's solution. Ultra-pure and clean platinum wires $(0.4 \mathrm{~mm})$ were used to connect the electrodes and input pin of the amplifier to prevent any electrolysis potential. The EAD signals of both antennae were fed to an HP 34465A digital multimeter (Keysight, Santa Rosa, USA) in DC measuring mode at 10 reads per second. Signal data were recorded using BenchVue software (Keysight). In GC-EAD analyses, an HP7890B GC (Agilent) was used to analyse the CHC solvent extracts with $1.0 \mathrm{~mL} / \mathrm{min}$ helium as carrier gas. The splitless injector was heated to $280{ }^{\circ} \mathrm{C}$. An HP-5 (30 m $\times 320 \mu \mathrm{m}$ $\times 0.32 \mu \mathrm{m}$ film, Agilent) column was used. The oven ramp was set at $200{ }^{\circ} \mathrm{C}$ for 1 min and then $10{ }^{\circ} \mathrm{C} / \mathrm{min}$ to $280{ }^{\circ} \mathrm{C}$ for $10 \mathrm{~min}$. The EAD transfer line was heated to $280{ }^{\circ} \mathrm{C}$ to prevent condensation of CHCs. The column effluents were diluted by a 42 $\mathrm{cm} / \mathrm{s}$ clean, humidified, static-free (filter by grounded copper mesh) air flow in a PTFE odour pipette ( $8.0 \mathrm{~mm}$ in diameter) before being delivered to the antennal preparation. One bee equivalent of CHCs in $2 \mu \mathrm{L}$ hexane was injected for each test. The GC-EAD tests were repeated 9 times. Only reproducible responses in all samples were considered EAD active responses.

\section{CHC vapour pressure calculations}

Vapour pressures (VP) of CHCs were calculated using the MPBPVP module in EPI Suite 4.11 (EPA, New York, USA). Three methods were used for calculation, the Antoine method (8), Modified Grain method $^{9}$, and Mackay method (9). The ratio of $V P s$ at high and low temperatures, including $V P_{45^{\circ} \mathrm{C}} / V P_{15^{\circ} \mathrm{C}}$ and $V P_{45^{\circ} \mathrm{C}} / V P_{25^{\circ} \mathrm{C}}$ were calculated and plotted for comparison. For consistency, no experimental data were used in calculation.

\section{Statistical analysis}

The effects of samples, colonies, and environment temperature on the counts of samples removed/tested were analysed using a Generalized Linear Mixed Model (GLMM) with Binary Probit (for dissections test and heated and/or washed dead bee test) or Poisson Loglinear (for wax concentration test) as link function. Estimated marginal means were compared using the Sequential Bonferroni method. Mean 
1 temperatures of active living bees were estimated from the sampled bees using a $t$-test.

2 The differences of compound quantities in dead and living bees were compared using

3 a paired-sample t-test. In the synthetic CHC assays, the effect of sample, colony, and

4 environmental temperature on the times of dead bee removal were analysed using a

5 Generalized Linear Model (GLM). The estimated marginal means (EM: mean \pm error)

6 were compared using the Sequential Bonferroni method.

7

8 
bioRxiv preprint doi: https://doi.org/10.1101/2020.03.05.978262; this version posted March 5, 2020. The copyright holder for this preprint (which was not certified by peer review) is the author/funder, who has granted bioRxiv a license to display the preprint in perpetuity. It is made available under aCC-BY-NC-ND 4.0 International license.

\section{Supplementary Figures and Table}

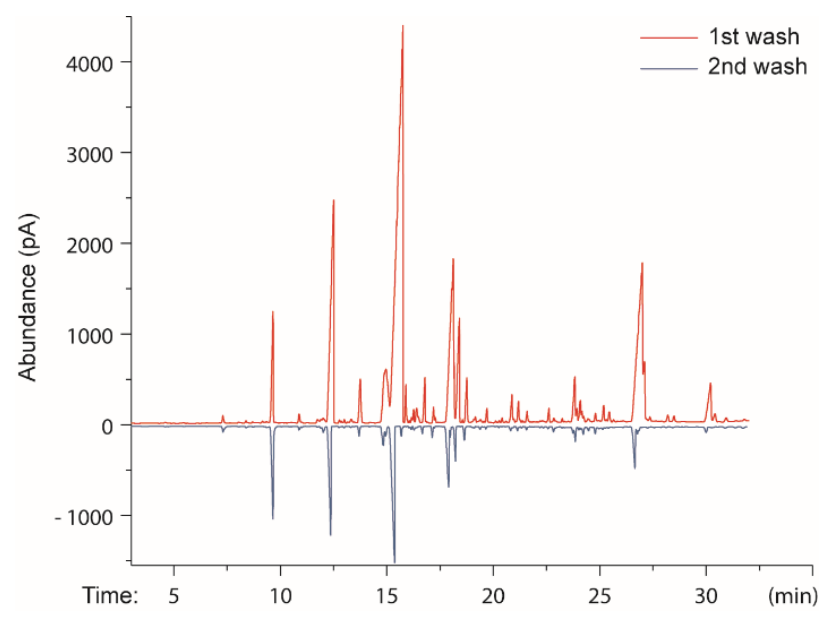

3 Figure S1 Solvent wash reduced not eliminated the $\mathrm{CHC}$

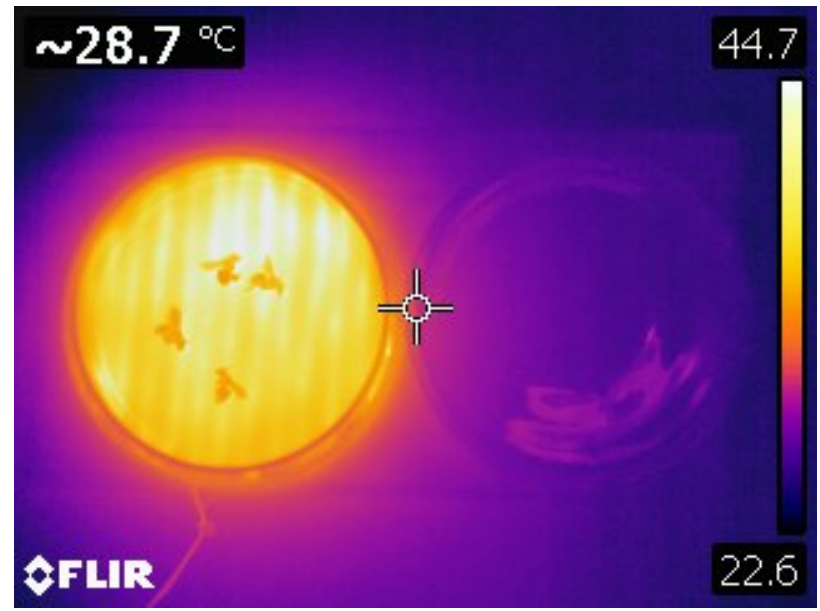

$5 \quad$ Figure S2 Thermo image of the heating apparatus

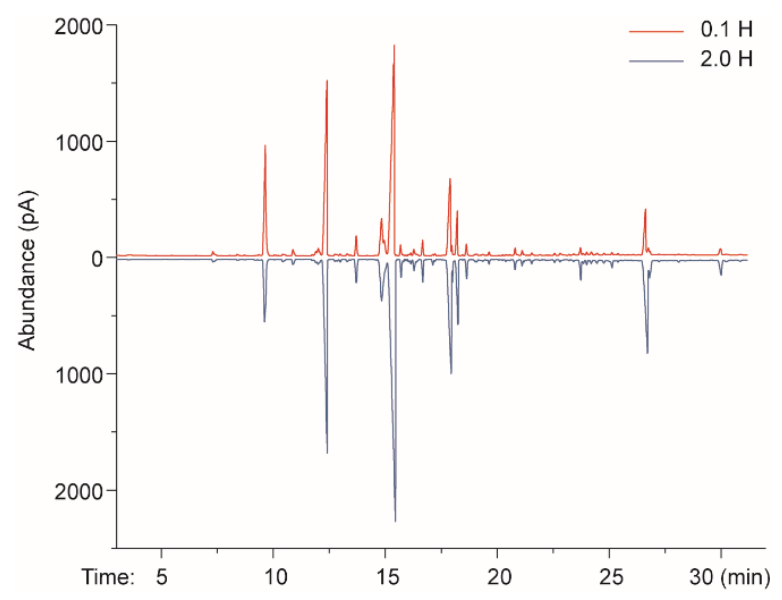

7 Figure $\mathrm{S} 3 \mathrm{CHC}$ residue did not change fast with time after death 

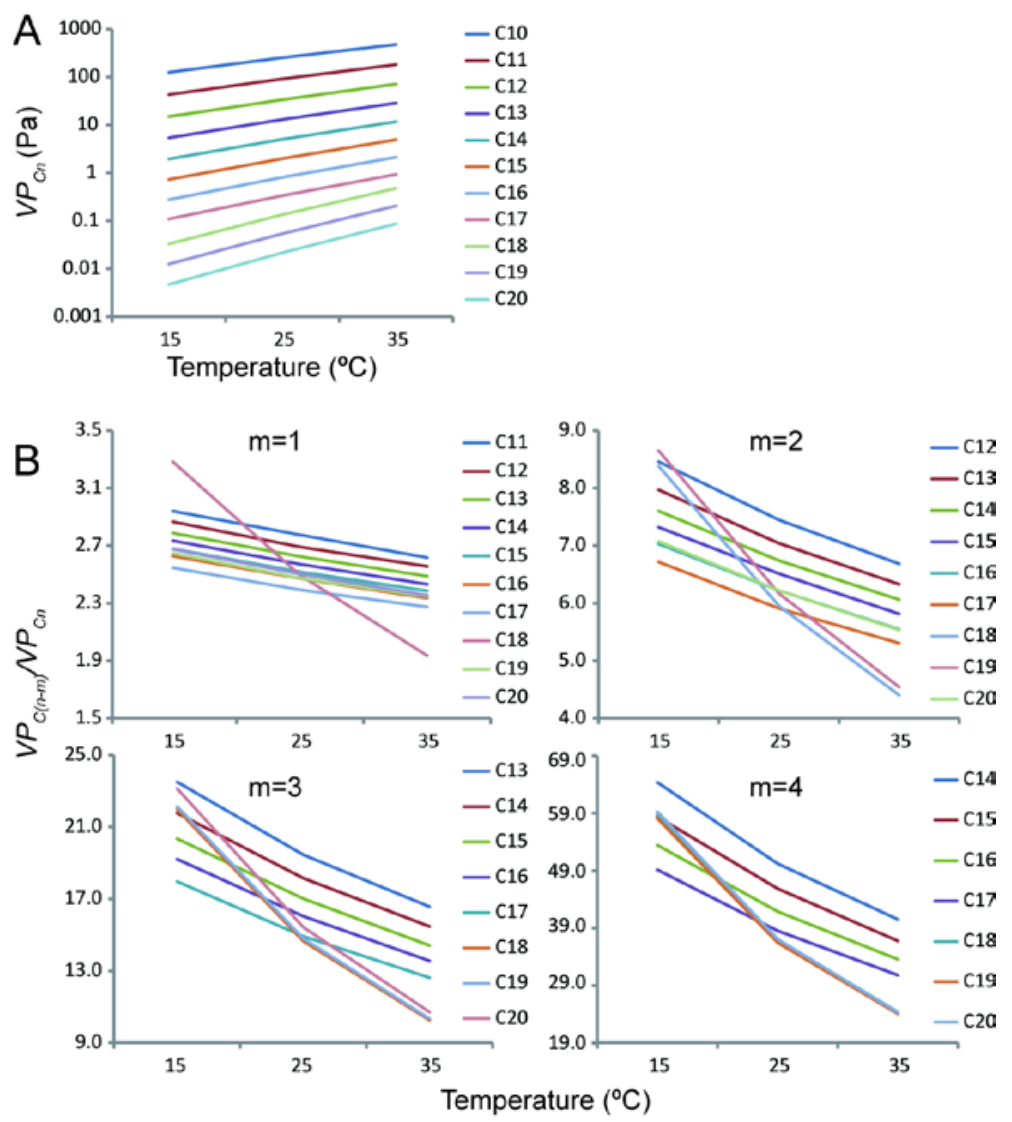

1

2

3

5

6

Figure S4 Temperature change the ratio of hydrocarbons vapour pressure

A Calculated vapour pressure of C10-C20 n-alkanes using Antoine method. B Changed VP ratio between the alkanes with 1, 2, 3, 4 carbon differences in molecule sizes.

Table S1 Sizes of tested colonies

\begin{tabular}{ll}
\hline Colony No. & $\begin{array}{l}\text { Number of } \\
\text { individuals }\end{array}$ \\
\hline $\mathbf{1}$ & $500^{\sim} 1000$ \\
$\mathbf{2}$ & $3000^{\sim} 3500$ \\
$\mathbf{3}$ & $2000^{\sim} 3000$ \\
$\mathbf{4}$ & $3500^{\sim} 4000$ \\
$\mathbf{5}$ & $5000^{\sim} 6000$ \\
\hline
\end{tabular}

Supplementary references

1. Tulloch AP. Beeswax-Composition and Analysis. Bee World. 1980;61(2):47-62.

2. Fröhlich B, Tautz J, Riederer M. Chemometric classification of comb and cuticular waxes of the honeybee Apis mellifera carnica. J Chem Ecol. 2000;26(1):123-37.

3. Van Oystaeyen A, Oliveira RC, Holman L, van Zweden JS, Romero C, Oi CA, et al. Conserved class of queen pheromones stops social insect workers from reproducing. Science. 2014;343(6168):287-90. 4. Rahman S, Hajong SR, Gévar J, Lenoir A, Darrouzet E. Cuticular hydrocarbon compounds in worker castes and their role in nestmate recognition in Apis cerana indica. J Chem Ecol.

2016;42(5):444-51. 
1 5. Thom C, Gilley DC, Hooper J, Esch HE. The scent of the waggle dance. Plos Biol. 2007;5(9):e228.

2 6. Wen P, Cheng Y-N, Qu Y-F, Zhang H-X, Li J-J, Bell H, et al. Foragers of sympatric Asian honey bee

3 species intercept competitor signals by avoiding benzyl acetate from Apis cerana alarm pheromone.

4 Sci Rep-Uk. 2017;7(1):6721.

5 7. Wen P, Cheng Y-N, Dong S-H, Wang Z-W, Tan K, Nieh JC. The sex pheromone of a globally invasive

6 honey bee predator, the Asian eusocial hornet, Vespa velutina. Sci Rep-Uk. 2017;7(1):12956.

7 8. Lyman WJ, ReehI WF, Rosenblatt DH. Handbook of chemical property estimation methods.

8 United States: Washington, DC (USA); American Chemical Society; 1990 1990-01-01.

9 9. Lyman WJ. Estimation of physical properties. In: Neely WB, Blau GE, editors. Environmental

10 exposure from chemicals. 1. Boca Raton: CRC Press; 1985. 